hep-th/9711136

JINR-E2-98-84

\title{
TENSIONLESS BRANES AND THE NULL STRING CRITICAL DIMENSION $\mathrm{G}$
}

\author{
P. Bozhilov 目 \\ Bogoliubov Laboratory of Theoretical Physics, JINR, Dubna, Russia \\ E-mail: bojilov@thsun1.jinr.dubna.su
}

BRST quantization is carried out for a model of $p$-branes with second class constraints. After extension of the phase space the constraint algebra coincides with the one of null string when $p=1$. It is shown that in this case one can or can not obtain critical dimension for the null string, depending on the choice of the operator ordering and corresponding vacuum states. When $p>1$, operator orderings leading to critical dimension in the $p=1$ case are not allowed. Admissable orderings give no restrictions on the dimension of the embedding space-time. Finally, a generalization to supersymmetric null branes is proposed.

\footnotetext{
${ }^{1}$ Work supported in part by the National Science Foundation of Bulgaria under contract $\phi-620 / 1996$

${ }^{2}$ permanent address: Dept.of Theoretical Physics,"Konstantin Preslavsky" Univ. of Shoumen 9700 Shoumen, Bulgaria E-mail: bojilov@uni-shoumen.bg
} 


\section{Introduction}

Recently a lot of papers are devoted to the tensionless (null) strings and their application in different areas and different dimensions [1]. In connection with the above activity it is worth to consider again the question about the critical dimension of the null strings and more generally - null $p$-branes. There are two answers to this question in the literature. Most of the authors insist on the nonexistence of critical dimension for such objects [2], but some other receive opposite results [3]. In our opinion the reason is in the different approaches to the choice of the operator ordering. If one looks at the classical null string as a collection of particles moving under certain conditions and wants to keep this picture in the quantum case also, there is no reason to expect a critical dimension emerging. Therefore one adopts such operator ordering which supports this point of view. If one does not bother about previous particle interpretation but simply compares the appearance of the anomaly in the constraint algebras of the null and usual string upon quantization (with one and the same operator ordering), one sees that the non-trivial central terms arise independently of the string tension $T$. Then the existence of critical dimension for the tensionless string is not surprising at all. On the other hand, it can be shown, that in the quantum Virasoro algebra of the usual string the limit $T ß 0$ can be taken consistently to obtain the null string gauge algebra with a vanishing critical dimension [4]. So what is the correct answer to the question about the existence of critical dimension for the null string? In this article we propose a pure technical resolution of the problem. The right operator ordering is that, which can be applied to higher dimensions, i.e. to null $p$-branes too. In our case we find two such orderings and they lead to the absence of critical dimensions for the null $p$-branes $(p \geq 1)$.

Here we quantize a model of $p$-branes [5] which initially do not describe null strings (when $p=1$ ), because the constraints are second class. However, it turns out that at the quantum level the constraint algebra coincides with one of the tensionless string. Checking quantum consistency of the theory for four different operator orderings we find $D=26$ for the critical dimension of the bosonic null string when "string-like" and Weyl orderings are applied. However, we do not receive any condition on the space-time dimension when apply "particle-like" and normal ordering. Investigating the case $p>1$, we observe that the first two orderings are forbidden by the Jacobi identity. Adopting the last two types of ordering, we reach to the conclusion that tensionless $p$-branes have no critical dimension for $p>1$. Because these orderings also apply to the case $p=1$, this conclusion is valid for all $p=1,2, \ldots$.

The paper is organized as follows. In section $\mathbf{2}$ we deal with the classical theory. With the help of the BRST charge, we construct BRST invariant hamiltonian and also give the corresponding Lagrangian. Then we solve the classical equations of motion and obtain the onshell expressions for the BRST charge $Q$ and for the constraints. Assuming periodic boundary conditions, we rewrite all quantities in Fourier modes. Section $\mathbf{3}$ is devoted to the quantization of the model. We define the renormalized operators and investigate the anomalies in the quantum constraint algebra. As a result, we obtain the conditions for quantum consistency of 
the theory for different values of $p$. In section 4 we propose a supersymmetric extension of the model under consideration which can be used to describe tensionless super $p$-branes. Finally, in section $\mathbf{5}$ we give some comments and conclusions.

\section{Classical theory}

To begin with, we first write down the hamiltonian of the classical model of $p$-branes with second class constraints [6] proposed in [5]. It can be cast in the form [7]:

$$
H_{0}=\int d^{p} \sigma\left(\lambda^{0} \psi_{0}+\lambda^{a} \psi_{a}\right), \quad a=1, \ldots, p
$$

where $\lambda^{0}, \lambda^{a}$ are Lagrange multipliers being arbitrary functions of the time parameter $\tau$ and volume coordinates $\sigma_{1}, \ldots, \sigma_{p}$. The constraints $\psi_{0}, \psi_{a}$ are defined by the equalities:

$$
\begin{array}{r}
\psi_{0}=p^{\alpha} p_{\alpha}+T^{2} \quad, \quad \psi_{a}=\eta_{\alpha \beta} p^{\alpha} \partial_{a} x^{\beta}, \\
\alpha, \beta=0,1, \ldots, D-2, \quad \eta_{\alpha \beta}=\operatorname{diag}(-1,1, \ldots, 1)
\end{array}
$$

Here $x^{\alpha}$ and $p_{\alpha}$ are canonically conjugated coordinates and momenta, $\partial_{a}=\partial / \partial \sigma^{a}, T=$ const.

The algebra of the constraints (2) is given by the Poisson bracket relations

$$
\begin{aligned}
& \left\{\psi_{0}\left(\underline{\sigma_{1}}\right), \psi_{0}\left(\underline{\sigma_{2}}\right)\right\}=0 \\
& \left\{\psi_{0}\left(\underline{\sigma_{1}}\right), \psi_{a}\left(\underline{\sigma_{2}}\right)\right\}=\left[\psi_{0}\left(\underline{\sigma_{1}}\right)+\psi_{0}\left(\underline{\sigma_{2}}\right)-2 T^{2}\right] \partial_{a} \delta^{p}\left(\underline{\sigma_{1}}-\underline{\sigma_{2}}\right), \\
& \left\{\psi_{a}\left(\underline{\sigma_{1}}\right), \psi_{b}\left(\underline{\sigma_{2}}\right)\right\}=\left[\delta_{a}^{c} \psi_{b}\left(\underline{\sigma_{1}}\right)+\delta_{b}^{c} \psi_{a}\left(\underline{\sigma_{2}}\right)\right] \partial_{c} \delta^{p}\left(\underline{\sigma_{1}}-\underline{\sigma_{2}}\right),
\end{aligned}
$$

where the notation $\underline{\sigma}=\left(\sigma_{1}, \ldots, \sigma_{p}\right)$ is used. It follows from here that the constraints are second class.

Introducing the hamiltonian (1), one has to check the consistency conditions [6]

$$
\left\{\psi_{0}, H_{0}\right\} \approx 0 \quad, \quad\left\{\psi_{a}, H_{0}\right\} \approx 0
$$

where $\approx$ denotes weak equality, i.e. equality up to constraints. In the present case these conditions are

$$
\partial_{a} \lambda^{0}=0 \quad, \quad \partial_{a} \lambda^{a}=0
$$

One of the methods for quantization of dynamical systems with second class constraints consists in passing to a system with first class constraints only [8], and then perform the quantization. To achieve this in our case, we enlarge the initial phase space with a new canonical pair $\left(x_{D-1}, p_{D-1}\right)$. This allows for transition from initial constraints $(2)$ to the new ones [9], 10]:

$$
\begin{aligned}
\varphi_{0} & =p^{\mu} p_{\mu}=p^{\alpha} p_{\alpha}+p_{D-1}^{2} \\
\varphi_{a} & =\eta_{\mu \nu} p^{\mu} \partial_{a} x^{\nu}=\eta_{\alpha \beta} p^{\alpha} \partial_{a} x^{\beta}+p_{D-1} \partial_{a} x_{D-1}
\end{aligned}
$$

$\varphi_{0}$ and $\varphi_{a}$ obey the Poisson bracket algebra

$$
\begin{aligned}
& \left\{\varphi_{0}\left(\underline{\sigma_{1}}\right), \varphi_{0}\left(\underline{\sigma_{2}}\right)\right\}=0, \\
& \left\{\varphi_{0}\left(\underline{\sigma_{1}}\right), \varphi_{a}\left(\underline{\sigma_{2}}\right)\right\}=\left[\varphi_{0}\left(\underline{\sigma_{1}}\right)+\varphi_{0}\left(\underline{\sigma_{2}}\right)\right] \partial_{a} \delta^{p}\left(\underline{\sigma_{1}}-\underline{\sigma_{2}}\right), \\
& \left\{\varphi_{a}\left(\underline{\sigma_{1}}\right), \varphi_{b}\left(\underline{\sigma_{2}}\right)\right\}=\left[\delta_{a}^{c} \varphi_{b}\left(\underline{\sigma_{1}}\right)+\delta_{b}^{c} \varphi_{a}\left(\underline{\sigma_{2}}\right)\right] \partial_{c} \delta^{p}\left(\underline{\sigma_{1}}-\underline{\sigma_{2}}\right),
\end{aligned}
$$


which means, that they are first class quantities. The corresponding hamiltonian is

$$
H=\int d^{p} \sigma\left(\mu^{0} \varphi_{0}+\mu^{a} \varphi_{a}\right)
$$

The Dirac consistency conditions

$$
\left\{\varphi_{0}, H\right\} \approx 0 \quad, \quad\left\{\varphi_{a}, H\right\} \approx 0
$$

do not place any restrictions on the Lagrange multipliers $\mu^{0}, \mu^{a}$.

Now, two notes are in order. The first one is that at any moment one can return to the initial dynamical system by dimensional reduction. The second is, that the algebra (3) of the constraints $\varphi_{0}, \varphi_{a}$ coincides with the tensionless limit of the usual $p$-brane ones [11]. That is why our conclusions about the critical dimensions, arising after quantization, will be also valid for the tensionless branes.

Following the BFV-BRST method for quantization of constrained systems [12], we now introduce for each constraint $\varphi_{0}, \varphi_{a}$ a pair of anticommuting ghost variables $\left(\eta^{0}, P_{0}\right),\left(\eta^{a}, P_{a}\right)$ respectively, which are canonically conjugated.Then the BRST charge is [13]

$$
Q=\int d^{p} \sigma\left\{\varphi_{0} \eta^{0}+\varphi_{a} \eta^{a}+P_{0}\left[\left(\partial_{a} \eta^{a}\right) \eta^{0}+\left(\partial_{a} \eta^{0}\right) \eta^{a}\right]+P_{b}\left(\partial_{a} \eta^{b}\right) \eta^{a}\right\}
$$

and it has the property

$$
\{Q, Q\}_{p b}=0
$$

where $\{., .\}_{p b}$ is the Poisson bracket in the extended phase space $\left(x^{\nu}, p_{\mu} ; \eta^{0}, P_{0} ; \eta^{a}, P_{b}\right)$.

In the new phase space, the constraints are given by the following brackets [14]:

$$
\begin{aligned}
\varphi_{0}^{t o t} & =\left\{Q, P_{0}\right\}_{p b}=\varphi_{0}+2 P_{0} \partial_{a} \eta^{a}+\left(\partial_{a} P_{0}\right) \eta^{a}=\varphi_{0}+\varphi_{0}^{g h} \\
\varphi_{a}^{t o t} & =\left\{Q, P_{a}\right\}_{p b}=\varphi_{a}+2 P_{0}\left(\partial_{a} \eta^{0}\right)+\left(\partial_{a} P_{0}\right) \eta^{0}+P_{a} \partial_{b} \eta^{b}+P_{b}\left(\partial_{a} \eta^{b}\right)+\left(\partial_{b} P_{a}\right) \eta^{b}=\varphi_{a}+\varphi_{a}^{g h}
\end{aligned}
$$

and they are first class. The BRST invariant hamiltonian is 12

$$
H_{\chi}=\{Q, \chi\}_{p b} \quad, \quad\left\{Q, H_{\chi}\right\}_{p b}=0,
$$

where $\chi$ is arbitrary, anticommuting, gauge fixing function. We choose

$$
\chi=\Lambda^{0} \int d^{p} \sigma P_{0}+\Lambda^{a} \int d^{p} \sigma P_{a}, \quad \Lambda^{0}, \Lambda^{a}-\text { const }
$$

and obtain:

$$
H_{\chi}=\int d^{p} \sigma\left[\Lambda^{0} \varphi_{0}^{t o t}+\Lambda^{a} \varphi_{a}^{t o t}\right]
$$

Let us note that additional set of canonically conjugated ghosts $\left(\bar{\eta}_{0}, \bar{P}^{0}\right),\left(\bar{\eta}_{a}, \bar{P}^{a}\right)$ must be added if we wish to write down the corresponding BRST invariant Lagrangian. If so, $Q$ and $\chi$ have to be modified in the following fashion [12, 14]

$$
\begin{gathered}
\tilde{Q}=Q+\int d^{p} \sigma\left(M_{0} \bar{P}^{0}+M_{a} \bar{P}^{a}\right) \\
\tilde{\chi}=\chi+\int d^{p} \sigma\left[\bar{\eta}_{0}\left(\chi^{0}+\frac{\rho_{1}}{2} M^{0}\right)+\bar{\eta}_{a}\left(\chi^{a}+\frac{\rho_{2}}{2} M^{a}\right)\right],
\end{gathered}
$$


where $M_{0}, M_{a}$ are the momenta, canonically conjugated to $\mu^{0}$ and $\mu^{a}$ respectively, $\chi^{0}$ and $\chi^{a}$ are gauge fixing conditions [15] for $\varphi_{0}$ and $\varphi_{a}, \rho_{1}$ and $\rho_{2}$ are parameters. All this results in the Lagrangian density $\left(\partial_{\tau}=\partial / \partial \tau\right)$ :

$$
L_{\tilde{\chi}}=L+L_{G F}+L_{G H},
$$

where

$$
L=\left(1 / 4 \mu^{0}\right)\left(\partial_{\tau} x-\mu^{a} \partial_{a} x\right)^{2},
$$

the gauge fixing part is

$$
L_{G F}=\frac{1}{2 \rho_{1}}\left(\partial_{\tau} \mu^{0}-\chi^{0}\right)\left(\partial_{\tau} \mu_{0}-\chi_{0}\right)+\frac{1}{2 \rho_{2}}\left(\partial_{\tau} \mu^{a}-\chi^{a}\right)\left(\partial_{\tau} \mu_{a}-\chi_{a}\right)
$$

and the ghost part is

$$
\begin{array}{r}
L_{G H}=-\partial_{\tau} \bar{\eta}_{0} \partial_{\tau} \eta^{0}-\partial_{\tau} \bar{\eta}_{a} \partial_{\tau} \eta^{a}+\mu^{0}\left[2 \partial_{\tau} \overline{\eta_{0}} \partial_{a} \eta^{a}+\left(\partial_{a} \partial_{\tau} \bar{\eta}_{0}\right) \eta^{a}\right] \\
+\mu^{a}\left[2 \partial_{\tau} \bar{\eta}_{0} \partial_{a} \eta^{0}+\left(\partial_{a} \partial_{\tau} \bar{\eta}_{0}\right) \eta^{0}+\partial_{\tau} \overline{\eta_{b}} \partial_{a} \eta^{b}+\partial_{\tau} \bar{\eta}_{a} \partial_{b} \eta^{b}+\left(\partial_{b} \partial_{\tau} \bar{\eta}_{a}\right) \eta^{b}\right] \\
+\int d^{p} \sigma^{\prime}\left\{\bar{\eta}_{0}\left(\sigma^{\prime}\right)\left[\left\{\varphi_{0}, \chi^{0}\left(\sigma^{\prime}\right)\right\}_{p b} \eta^{0}+\left\{\varphi_{a}, \chi^{0}\left(\sigma^{\prime}\right)\right\}_{p b} \eta^{a}\right]\right. \\
\left.+\bar{\eta}_{a}\left(\sigma^{\prime}\right)\left[\left\{\varphi_{0}, \chi^{a}\left(\sigma^{\prime}\right)\right\}_{p b} \eta^{0}+\left\{\varphi_{b}, \chi^{a}\left(\sigma^{\prime}\right)\right\}_{p b} \eta^{b}\right]\right\}
\end{array}
$$

Let us now go back to the hamiltonian picture. The hamiltonian (4) leads to equations of motion with the following general solution for the bosonic variables [5]

$$
\begin{aligned}
x^{\nu} & =y^{\nu}(\underline{z})+2 g(\tau) p^{\nu}(\underline{z}), \\
p_{\nu} & =p_{\nu}(\underline{z}),
\end{aligned}
$$

and for the ghosts [13]

$$
\begin{aligned}
\eta^{0} & =\zeta^{0}(\underline{z})+g(\tau) \partial_{a} \eta^{a}(\underline{z}), \\
P_{0} & =P_{0}(\underline{z}), \\
\eta^{a} & =\eta^{a}(\underline{z}), \\
P_{a} & =\Pi_{a}(\underline{z})+g(\tau) \partial_{a} P_{0}(\underline{z}) .
\end{aligned}
$$

Here $y^{\nu}, p_{\nu}, \zeta^{0}, P_{0}, \eta^{a}$ and $\Pi_{a}$ are arbitrary functions of the variables $z^{a}$,

$$
z^{a}=\Lambda^{a} \tau+\sigma^{a} \quad \text { and } \quad g(\tau)=\Lambda^{0} \tau .
$$

On the solutions (5) the BRST charge $Q$ takes the form [13]

$$
Q^{S}=\int d^{p} z\left\{\phi_{0} \zeta^{0}+\phi_{a} \eta^{a}+P_{0}\left[\left(\partial_{a} \eta^{a}\right) \zeta^{0}+\left(\partial_{a} \zeta^{0}\right) \eta^{a}\right]+\Pi_{b}\left(\partial_{a} \eta^{b}\right) \eta^{a}\right\},
$$

where $\phi_{0}=p^{2}(\underline{z}), \phi_{a}=p_{\nu}(\underline{z}) \partial_{a} y^{\nu}(\underline{z})$. Now the constraints are

$$
\phi_{0}^{\text {tot }}(\underline{z})=\left\{Q^{S}, P_{0}(\underline{z})\right\}_{p b} \quad, \quad \phi_{a}^{\text {tot }}(\underline{z})=\left\{Q^{S}, \Pi_{a}(\underline{z})\right\}_{p b},
$$

and they are connected with $\varphi_{0}^{t o t}, \varphi_{a}^{\text {tot }}$ by the equalities

$$
\varphi_{0}^{t o t}(\underline{z})=\phi_{0}^{t o t}(\underline{z}) \quad, \quad \varphi_{a}^{t o t}=\phi_{a}^{\text {tot }}(\underline{z})+g(\tau) \partial_{a} \phi_{0}^{\text {tot }}(\underline{z}) .
$$


From now on, we confine ourselves to the case of periodic boundary conditions when our phase-space variables admit Fourier series expansions. Let us denote the Fourier components of $y^{\nu}, p^{\nu}, \zeta^{0}, P_{0}, \eta^{a}$ and $\Pi_{a}$ with $x_{\underline{k}}^{\nu}, p_{\underline{k}}^{\nu}, c_{\underline{k}}, b_{\underline{k}}, \bar{c}_{\underline{k}}^{a}$ and $\bar{b}_{a, \underline{k}}$ respectively. For the zero modes of $p^{\nu}$ and $x^{\nu}$, we introduce the notations

$$
P^{\mu}=(2 \pi)^{p} p_{\underline{0}}^{\mu} \quad, \quad q^{\nu}=\frac{-i}{(2 \pi)^{p}} x_{\underline{0}}^{\nu} .
$$

Then we have the following non-zero Poisson brackets:

$$
\begin{aligned}
\left\{P^{\mu}, q^{\nu}\right\}_{p b} & =-\eta^{\mu \nu}, \\
\left\{p_{\underline{k}}^{\mu}, x_{\underline{n}}^{\nu}\right\}_{p b} & =-i \eta^{\mu \nu} \delta_{\underline{k}+\underline{n}, \underline{0}}, \\
\left\{c_{\underline{k}}, b_{\underline{n}}\right\}_{p b} & =-i \delta_{\underline{k}+\underline{n}, \underline{0}}, \\
\left\{\bar{c}_{\underline{k}}^{a}, \bar{b}_{b, \underline{n}}\right\}_{p b} & =-i \delta_{b}^{a} \delta_{\underline{k}+\underline{n}, \underline{0} .}
\end{aligned}
$$

The Fourier expansions for the constraints $\phi_{0}^{\text {tot }}$ and $\phi_{a}^{\text {tot }}$ are

$$
\phi_{0}^{t o t}(\underline{z})=\frac{1}{(2 \pi)^{p}} \sum_{\underline{m} \in Z^{p}} C_{\underline{m}}^{t o t} e^{-i \underline{m z}} \quad, \quad \phi_{a}^{t o t}(\underline{z})=\frac{1}{(2 \pi)^{p}} \sum_{\underline{m} \in Z^{p}} D_{a, \underline{m}}^{t o t} e^{-i \underline{m z}} .
$$

Here

$$
C_{\underline{n}}^{t o t}=i\left\{Q^{S}, b_{\underline{n}}\right\}_{p b}=C_{\underline{n}}+C_{\underline{n}}^{g h} \quad, \quad D_{a, \underline{n}}^{t o t}=i\left\{Q^{S}, \bar{b}_{a, \underline{n}}\right\}_{p b}=D_{a, \underline{n}}+D_{a, \underline{n}}^{g h}
$$

where

$$
\begin{aligned}
Q^{S} & =\sum_{\underline{n} \in Z^{p}}\left\{\left[C_{\underline{n}}+(1 / 2) C_{\underline{n}}^{g h}\right] c_{-\underline{n}}+\left[D_{a, \underline{n}}+(1 / 2) D_{a, \underline{n}}^{g h}\right] c_{-\underline{n}}^{a}\right\}, \\
C_{\underline{n}} & =(2 \pi)^{p} \sum_{\underline{k} \in Z^{p}} p_{\underline{k}}^{\nu} p_{\nu, \underline{n}-\underline{k}}, \\
D_{a, \underline{n}} & =-\sum_{\underline{k} \in Z^{p}}\left(n_{a}-k_{a}\right) p_{\underline{k}}^{\nu} x_{\nu, \underline{n}-\underline{k}}, \\
C_{\underline{n}}^{g h} & =\sum_{\underline{k} \in Z^{p}}\left(n_{a}-k_{a}\right) b_{\underline{n}+\underline{k}} \bar{c}_{-\underline{k}}^{a}, \\
D_{a, \underline{n}}^{g h} & =\sum_{\underline{k} \in Z^{P}}\left[\left(n_{a}-k_{a}\right) b_{\underline{n}+\underline{k}} c_{-\underline{k}}+\left(\delta_{a}^{c} n_{b}-\delta_{b}^{c} k_{a}\right) \bar{b}_{c, \underline{n}+\underline{k}} \bar{c}_{-\underline{k}}^{b}\right]
\end{aligned}
$$

Using expressions (6) to (8), one obtains that the algebra of the total generators (7) is given by

$$
\begin{aligned}
\left\{C_{\underline{n}}^{t o t}, C_{\underline{m}}^{t o t}\right\}_{p b} & =0 \\
\left\{C_{\underline{n}}^{t o t}, D_{a, \underline{m}}^{t o t}\right\}_{p b} & =-i\left(n_{a}-m_{a}\right) C_{\underline{n}+\underline{m}}^{t o t}, \\
\left\{D_{a, \underline{n}}^{t o t}, D_{b, \underline{m}}^{t o t}\right\}_{p b} & =-i\left(\delta_{a}^{c} n_{b}-\delta_{b}^{c} m_{a}\right) D_{c, \underline{n}+\underline{m}}^{t o t} .
\end{aligned}
$$

\section{Quantization}

Going to the quantum theory according to the rule $i\{., \cdot\}_{p b} \rightarrow$ (anti)commutator, we define $Q^{S}$ by introducing the renormalized operators $\left(\alpha, \beta_{a}-\right.$ const $)$

$$
C_{\underline{n}}^{t o t}=C_{\underline{n}}+C_{\underline{n}}^{g h}-\alpha \delta_{\underline{n}, \underline{0}} \quad, \quad D_{a, \underline{n}}^{t o t}=D_{a, \underline{n}}+D_{a, \underline{n}}^{g h}-\beta_{a} \delta_{\underline{n}, \underline{0}}
$$


and postulating [13]

$$
\begin{aligned}
Q^{S}= & \sum_{\underline{n} \in Z^{p}}:\left\{\left[C_{\underline{n}}+(1 / 2) C_{\underline{n}}^{g h}-\alpha \delta_{\underline{n}, \underline{0}}\right] c_{-\underline{n}}\right. \\
& \left.+\left[D_{a, \underline{n}}+(1 / 2) D_{a, \underline{n}}^{g h}-\beta_{a} \delta_{\underline{n}, \underline{0}}\right] \bar{c}_{-\underline{n}}^{a}\right\}:
\end{aligned}
$$

where :...: stands for operator ordering and in $C_{\underline{n}}, \ldots, D_{a, \underline{n}}^{g h}$ operator ordering is also assumed.

Let us turn to the question about the critical dimensions which might appear in the model under consideration. As is well known, the critical dimension arises as a necessary condition for nilpotency of the BRST charge operator. In turn, this is connected with the vanishing of the central charges in the quantum constraint algebra. Because of that, we are going to find out the central terms which appear in our quantum gauge algebra for different values of $p$ (the most general form of central extension, which is compatible with the Jacobi identities is written in the Appendix).

We start with the case $p=1$, which corresponds to a closed string. In this case $a=b=1$ and one defines the operator ordering with respect to $p_{-n}^{\nu}, \ldots, \bar{c}_{-n}$ and $p_{n}^{\nu}, \ldots, \bar{c}_{n},(n>0)$, so that

$$
p_{-n}^{\nu}\left|0>=\ldots=\bar{c}_{-n}\right| 0>=0 \quad, \quad<0\left|p_{n}^{\nu}=\ldots=<0\right| \bar{c}_{n}=0 .
$$

We call this ordering "string - like". Using the explicit expressions for the constraints (8), one obtains that central terms appear in the commutators $\left[D_{n}, D_{m}\right],\left[D_{n}^{g h}, D_{m}^{g h}\right]$ and they are respectively

$$
c=(D / 6)\left(n^{2}-1\right) n \delta_{n+m, 0} \quad, \quad c^{g h}=-(1 / 3)\left(13 n^{2}-1\right) n \delta_{n+m, 0} .
$$

Therefore, the quantum constraint algebra has the form

$$
\begin{aligned}
& {\left[C_{n}^{t o t}, C_{m}^{t o t}\right]=0} \\
& {\left[C_{n}^{t o t}, D_{m}^{\text {tot }}\right]=(n-m) C_{n+m}^{t o t}+2 \alpha n \delta_{n+m, 0},} \\
& {\left[D_{n}^{t o t}, D_{m}^{t o t}\right]=(n-m) D_{n+m}^{t o t}+(1 / 6)\left[(D-26) n^{2}+(12 \beta-D+2)\right] n \delta_{n+m, 0} .}
\end{aligned}
$$

This means that the conditions for the nilpotency of the BRST charge operator $Q^{S}$ are

$$
(D-26) n^{2}+(12 \beta-D+2)=0 \quad, \quad \alpha=0,
$$

which leads to the well known result $D=26, \beta=2$. Obviously, this reproduces one of the basic features of the quantized tensionful closed bosonic string - its critical dimension.

Going to the case $p>1$, one natural generalization of the creation and annihilation operators definition is

$$
p_{\underline{n}}^{\nu}\left|0>_{a}=0, \quad{ }_{a}<0\right| p_{-\underline{n}}^{\nu}=0, \quad \text { for } \quad \sum_{a=1}^{p} n_{a}>0
$$

and analogously for the operators $x_{\underline{n}}^{\nu}, \ldots, \bar{c}_{\underline{n}}^{a}$. However, it turns out that such definition does not agree with the Jacobi identities for the quantum constraint algebra (except for $p=1$ ). That is why, we introduce the creation $(+)$ and annihilation $(-)$ operators in the following way 13

$$
p_{\underline{n}}^{\nu}=(1 / \sqrt{2})\left(p_{\underline{n}}^{\nu+}+p_{-\underline{n}}^{\nu-}\right), \ldots, \bar{c}_{\underline{n}}^{a}=(1 / \sqrt{2})\left(\bar{c}_{\underline{n}}^{a+}+\bar{c}_{-\underline{n}}^{a-}\right)
$$


and respectively new vacuum states

$$
p_{\underline{n}}^{\nu-}\left|v a c>=\ldots=\bar{c}_{\underline{n}}^{a-}\right| v a c>=0 \quad, \quad<\operatorname{vac}\left|p_{\underline{n}}^{\nu+}=\ldots=<\operatorname{vac}\right| \bar{c}_{\underline{n}}^{a+}=0 .
$$

This choice of the creation and annihilation operators corresponds to the representation of all phase-space variables $p^{\nu}, \ldots, \bar{c}^{a}$ as sums of frequency parts which are conjugated to each other and satisfy the same equation of motion as the corresponding dynamical variable.

By direct computation one shows, that with operator product defined with respect to the introduced creation and annihilation operators (11) (we shall refer to as "normal ordering"), the central extension of the algebra of the gauge generators (10) does not appear, i.e. $\alpha=$ $0, \beta_{a}=0$. Consequently, the BRST charge operator $Q^{S}$ is automatically nilpotent in this case and there is no restriction on the dimension of the background space-time for $p>1$.

The impossibility to introduce a string - like operator ordering when $p>1$ leads to the problem of finding those operator orderings which are possible for $p=1$ as well as for $p>1$. First of all, we check the consistency of the (already used for $p>1$ ) normal ordering for $p=1$. It turns out that it is consistent, but now critical dimension for the null string does not appear. The same result - absence of critical dimension for every value of $p$, one obtains when uses the so called particle - like operator ordering. Now the ket vacuum is annihilated by momentum-type operators and the bra vacuum is annihilated by coordinate-type ones:

$$
\begin{aligned}
& p_{\underline{n}}^{\mu}\left|0>_{M}=b_{\underline{n}}\right| 0>_{M}=\bar{b}_{\underline{n}} \mid 0>_{M}=0, \\
& C<0\left|x_{\underline{n}}^{\mu}=C<0\right| c_{\underline{n}}={ }_{C}<0 \mid \bar{c}_{\underline{n}}=0 \quad, \quad \forall \underline{n} \in Z^{p} .
\end{aligned}
$$

Further, we check the case when Weyl ordering is applied. Now it turns out, that in the null string case $(p=1)$ this leads to critical dimension $D=26$, but for the null brane $(p>1)$ this ordering is inconsistent, as was the string - like one.

As a final result, we have four type of operator orderings checked. Two of them are valid for the string as well as for the brane and then we do not receive any critical dimension. The other two type of ordering give critical dimension $D=26$ for the string and are not applicable for the brane. Our opinion is that the right operator ordering is the one applicable for all $p=1,2, \ldots$. In other words, our viewpoint is that neither null strings nor null branes have critical dimensions. The same point of view is presented in [16.

Let us spend some more words about the impossibility to introduce at $p>1$ an operator ordering which at $p=1$ gives critical dimension. This is connected with the fact that the constraint algebra, as is shown in the Appendix, does not possess non-trivial central extension when $p>1$ (see also [7], [16]). As a matter of fact, the string critical dimension appears in front of $n^{3}$, i.e. in the non-trivial part of the constraint algebra central extension, which can not be taken away by simply redefining the generators $D_{n}$, in contrast to the trivial part $\sim n$. Because of the nonexistence of non-trivial central extension when $p>1$, any critical dimension arising is impossible in view of the Jacobi identities. Therefore, if the quantum null brane constraint algebra is given by (up to trivial central extensions)

$$
\begin{aligned}
{\left[C_{\underline{n}}^{t o t}, C_{\underline{m}}^{t o t}\right] } & =0 \\
{\left[C_{\underline{n}}^{t o t}, D_{a, \underline{m}}^{t o t}\right] } & =\left(n_{a}-m_{a}\right) C_{\underline{n}+\underline{m}}^{t o t}, \\
{\left[D_{a, \underline{n}}^{t o t}, D_{b, \underline{m}}^{t o t}\right] } & =\left(\delta_{a}^{c} n_{b}-\delta_{b}^{c} m_{a}\right) D_{c, \underline{n}+\underline{m}}^{t o t}
\end{aligned}
$$

then the latter has no critical dimension and exists in any D-dimensional space-time, when embedding of the $p+1$-dimensional worldvolume of the $p$-brane is possible there. 
Finally, we pay attention to the fact that in every one of the $p$ subalgebras (at fixed $a$ ) of the constraint algebra, one can obtain non-trivial central extension and consequently - critical dimension (see Appendix). For example, taking string - like or Weyl ordering, one derives $D=25+p$, which appears to be critical dimension for the tensile $p$-brane [17], [16]. However, the considered quantum dynamical system is described by the full constraint algebra, where only trivial central extensions are possible.

\section{Supersymmetrization}

It turns out that the model described in the previous sections can be generalized to include also spinorial degrees of freedom. This generalization is not straightforward, but the resulting dynamical system may be viewed as generated by its bosonic part, which in terms of constraints is equivalent to a system with Poisson bracket relations, given by (9), i.e. equivalent to the null bosonic brane. This new model possesses space-time supersymmetry and is characterized by the following classical first class constraints

$$
\begin{aligned}
\left\{T_{0}\left(\underline{\sigma}_{1}\right), T_{0}\left(\underline{\sigma}_{2}\right)\right\} & =0 \\
\left\{T_{0}\left(\underline{\sigma}_{1}\right), T_{\alpha}^{A}\left(\underline{\sigma}_{2}\right)\right\} & =0, \\
\left\{T_{0}\left(\underline{\sigma}_{1}\right), T_{a}^{A}\left(\underline{\sigma}_{2}\right)\right\} & =\left[T_{0}\left(\underline{\sigma}_{1}\right)+T_{0}\left(\underline{\sigma}_{2}\right)\right] \partial_{a} \delta^{p}\left(\underline{\sigma}_{1}-\underline{\sigma}_{2}\right), \\
\left\{T_{a}^{A}\left(\underline{\sigma}_{1}\right), T_{b}^{B}\left(\underline{\sigma}_{2}\right)\right\} & =\delta^{A B}\left[\delta_{a}^{c} T_{b}^{B}\left(\underline{\sigma}_{1}\right)+\delta_{b}^{c} T_{a}^{A}\left(\underline{\sigma}_{2}\right)\right] \partial_{c} \delta^{p}\left(\underline{\sigma}_{1}-\underline{\sigma}_{2}\right), \\
\left\{T_{a}^{A}\left(\underline{\sigma}_{1}\right), T_{\alpha}^{B}\left(\underline{\sigma}_{2}\right)\right\} & =\delta^{A B}\left[T_{\alpha}^{A}\left(\underline{\sigma}_{1}\right)+T_{\alpha}^{A}\left(\underline{\sigma}_{2}\right)\right] \partial_{a} \delta^{p}\left(\underline{\sigma}_{1}-\underline{\sigma}_{2}\right), \\
\left\{T_{\alpha}^{A}\left(\underline{\sigma}_{1}\right), T_{\beta}^{B}\left(\underline{\sigma}_{2}\right)\right\} & =-2 i \delta^{A B} \hat{P}_{\alpha \beta} T_{0}\left(\underline{\sigma}_{1}\right) \delta^{p}\left(\underline{\sigma}_{1}-\underline{\sigma}_{2}\right), \\
\hat{P}_{\alpha \beta} & =P_{\mu} \sigma_{\alpha \beta}^{\mu} .
\end{aligned}
$$

Comparing the above equalities with the spinning string and superstring constraint algebras, we conclude that they can be regarded as possible tensionless limit of the super $p$-brane case. However, this supersymmetric model will be considered in detail in a separate paper. Here we only note, that Poisson brackets in (12) give the naive version of the constraint algebra. Actually, there is a set of generators with which (12) must be enlarged.

\section{Comments and Conclusions}

In this paper we present the results on the quantization of the restricted $p$-brane [5] reported in [13]. On the other hand, we investigate the connection between the appearance of critical dimensions and different operator orderings for $p=1,2, \ldots$.

The quantization of the restricted $p$-brane in [13] is alternative to the one given in [10]. The latter is based on a previous work [9] on the quantization of the restricted string and treat asymmetrically the constraints $p^{\nu} \partial_{a} x_{\nu}=0$ for $a=1$ and $a=2,3, \ldots, p$. In [13] and here, we consider all these constraints on equal footing.

The observation, that there is an operator ordering which is valid $\forall p \in Z_{+}$and another one, which is admissable only for $p=1$ [13], leads to the problem of finding those orderings which are possible for every positive integer value of $p$. We applied here four types of operator orderings and we establish that two of them (normal ordering and particle-like ordering) are admissable $\forall p \in Z_{+}$, but the other two (string - like and Weyl ordering) are admissable only for $p=1$. The fact, that the latter two orderings lead to appearance of critical dimension, 
and the former two do not, is a consequence of the constraint algebra property to have nontrivial central extension only for $p=1$. On the other hand, the obtained nontrivial central extensions of the Virasoro type for some of it subalgebras, provide an explanation why the critical dimensions $D=25+p, p=1,2, \ldots$ [17], [16], re-derived also here, can emerge. However, our claim is, that the critical dimensions appearing in the subalgebras, must not be considered as such for the given model as a whole. The model is represented by the full constraint algebra, which does not possess non-trivial central extension for $p \geq 2$.

Since after BFV-BRST quantization our constraint algebra coincides with the null tension limit of the usual $p$-brane algebra [11], we deduce that the upper conclusions are valid for the tensionless $p$-branes also. This lead us to the proposition of the rule: the right operator orderings in the case of null string $(p=1)$ are those, which are admissable in the $p>1$ case too.

\section{Acknowledgments}

The author would like to thank A. Pashnev, M. Stoilov and D. Stoyanov for the useful discussions, and B. Dimitrov for critical reading of the manuscript. It is also a pleasure to thank F. Lizzi, A. Nicolaidis, P. Porfyriadis and P. Saltsidis for the given information about their related papers.

\section{Appendix}

Here we briefly comment on the possible central extensions of the algebra, given by the commutators:

$$
\begin{aligned}
{\left[A_{\underline{n}}, A_{\underline{m}}\right] } & =0, \\
{\left[A_{\underline{n}}, B_{a, \underline{m}}\right] } & =\left(n_{a}-m_{a}\right) A_{\underline{n}+\underline{m}}, \\
{\left[B_{a, \underline{n}}, B_{b, \underline{m}}\right] } & =\left(\delta_{a}^{c} n_{b}-\delta_{b}^{c} m_{a}\right) B_{c, \underline{n}+\underline{m}} \quad, \quad(a, b=1,2, \ldots, p) .
\end{aligned}
$$

To begin with, we modify the right hand sides of the upper equalities in the following way:

$$
\begin{aligned}
{\left[A_{\underline{n}}, A_{\underline{m}}\right] } & =d(\underline{n}, \underline{m}) \\
{\left[A_{\underline{n}}, B_{a, \underline{m}}\right] } & =\left(n_{a}-m_{a}\right) A_{\underline{n}+\underline{m}}+d_{a}(\underline{n}, \underline{m}), \\
{\left[B_{a, \underline{n}}, B_{b, \underline{m}}\right] } & =\left(\delta_{a}^{c} n_{b}-\delta_{b}^{c} m_{a}\right) B_{c, \underline{n}+\underline{m}}+d_{a b}(\underline{n}, \underline{m}) .
\end{aligned}
$$

Checking the Jacobi identities, involving the triplets $(A, A, B),(A, B, B)$ and $(B, B, B)$,one shows that there are only trivial solutions for $d(\underline{n}, \underline{m}), d_{a}(\underline{n}, \underline{m})$ and $d_{a b}(\underline{n}, \underline{m})$. Namely,

$$
\begin{aligned}
d(\underline{n}, \underline{m}) & =0 \quad, \quad d_{a}(\underline{n}, \underline{m})=\left(n_{a}-m_{a}\right) f(\underline{n}+\underline{m}), \\
d_{a b}(\underline{n}, \underline{m}) & =\left(\delta_{a}^{c} n_{b}-\delta_{b}^{c} m_{a}\right) g_{c}(\underline{n}+\underline{m}),
\end{aligned}
$$

where $f, g_{a}$ are arbitrary functions of their arguments. In particular, there exist the solutions

$$
\begin{aligned}
d_{a}(\underline{n}, \underline{m}) & =2 \alpha n_{a} \delta_{\underline{n}+\underline{m}, \underline{0}} \quad, \quad \alpha=\text { const }, \\
d_{a b}(\underline{n}, \underline{m}) & =\left(\beta_{a} n_{b}+\beta_{b} n_{a}\right) \delta_{\underline{n}+\underline{m}, \underline{0}} \quad, \quad \beta_{a}=\text { const },
\end{aligned}
$$

which might appear because of the operator ordering in $A_{\underline{n}}$ and $B_{a, \underline{n}}$. However, there are $p$ subalgebras with non-trivial central extensions (no summation over $\bar{a}$ ):

$$
\left[A_{\underline{n}}, A_{\underline{m}}\right]=0
$$




$$
\begin{aligned}
{\left[A_{\underline{n}}, B_{a, \underline{m}}\right] } & =\left(n_{a}-m_{a}\right) A_{\underline{n}+\underline{m}}+\left(q_{a} n_{a}^{2}+r_{a}\right) n_{a} \delta_{\underline{n}+\underline{m}, \underline{0}}, \\
{\left[B_{a, \underline{n}}, B_{a, \underline{m}}\right] } & =\left(n_{a}-m_{a}\right) B_{a, \underline{n}+\underline{m}}+\left(s_{a} n_{a}^{2}+t_{a}\right) n_{a} \delta_{\underline{n}+\underline{m}, \underline{0}}, \quad q_{a}, r_{a}, s_{a}, t_{a}-\text { const. }
\end{aligned}
$$

When $p=1$, there is one such subalgebra and it coincides with the full algebra.

\section{References}

[1] H. de Vega, A. Nicolaidis, Phys. Lett. B295 (1992) 214;

O. Ganor, A. Hanani, Nucl. Phys. B474 (1996) 122, hep-th/9602120;

C. Lousto, N. Sanches, Phys. Rev. D54 (1996) 6399, gr-qc/9605015;

O. Ganor, Nucl. Phys. B489 (1997) 95, hep-th/9605201;

A. Hanani, I. Klebanov, Nucl. Phys. B482 (1996) 105, hep-th/9606136;

O. Ganor, hep-th/9607092;

K. Davis, hep-th/9609005;

P. Mayr, Nucl. Phys. B494 (1997) 489, hep-th/9610162;

M. Dabrowski, A. Larsen, Phys. Rev. D55 (1997) 6409, hep-th/9610243;

B. Jensen, U. Lindström, Phys. Lett. B398 (1997) 83, hep-th/9612213;

P. Porfyriadis, D. Papadopoulos, hep-th/9707183;

K. Ilienko, A. Zheltukhin, hep-th/9708024.

[2] F. Lizzi, B. Rai, G. Sparano, A. Srivastava, Phys. Lett. B182 (1986) 326;

A. Karlhede, U. Lindström, Class. Quant. Grav. 3 (1986) L73;

R. Amorim, J. Barcelos-Neto, Z. Phys. C38 (1988) 643;

J. Barcelos-Neto, M. Ruiz-Altaba, Phys. Lett. B228 (1989) 193;

I. Bandos, A. Zheltukhin, Sov. J. Nucl. Phys. 50 (1989) 556;

H. de Vega, I. Giannakis, A. Nicolaidis, Mod. Phys. Lett. A10 (1995) 2479, hepth/9412081.

[3] J. Gamboa, C. Ramirez, M. Ruiz-Altaba, Phys. Lett. B225 (1989) 335;

R. Amorim, J. Barcelos-Neto, Phys. Lett. 253 (1991) 313.

[4] F. Lizzi, Mod. Phys. Lett. A9 (1994) 1495, hep-th/9404148;

A. Nicolaidis, J. Paschalis, P. Porfyriadis, hep-th/9702185.

[5] D. Stoyanov, Mod. Phys. Lett. A4 (1989) 1287.

[6] P. A. M. Dirac, Lectures on Quantum Mechanics (Yeshiva University, New York, 1964).

[7] M. Stoilov, D. Stoyanov, J. Phys. A23 (1990) 5925.

[8] L. Faddeev, S. Shatashvili, Phys. Lett. B167 (1986) 225;

E. Egoryan, R. Manvelyan, Theor. Math. Phys. 94 (1993) 241. 
[9] P. Bozhilov, M. Stoilov, Phys. Lett. B293 (1992) 335.

[10] M. Stoilov, Phys. Lett. B316 (1993) 80;

[11] B. Barbashov and V. Nesterenko, Introduction to the relativistic string theory, (World Scientific, Singapore, 1990);

[12] E. Fradkin, G. Vilkovisky, Phys. Lett. B55 (1975) 224;

I. Batalin, G. Vilkovisky, Phys. Lett. B69 (1977) 309;

E. Fradkin, T. Fradkina, Phys. Lett. B72 (1978) 343;

M. Henneaux, Phys. Rep. 126 (1985) 1.

[13] P. Bozhilov, BRST quantization of a bosonic $p$-brane model, in: Proc. of $25^{\text {th }}$ Anniversary Conference of the "Konstantin Preslavsky" Univ. of Shoumen, 30.10-02.11.1996, Shoumen, Bulgaria.

[14] M. Henneaux, Phys. Lett. B120 (1983) 179;

K. Fujikawa, J. Kubo, Phys. Lett. B199 (1987) 75.

[15] R. Marnelius, Phys. Lett. B99 (1981) 467;

R. Marnelius, Acta Phys. Polon. B13 (1982) 669.

[16] P. Saltsidis, Phys. Lett. B401 (1997) 21, hep-th/9702081;

[17] U. Marquard, M. Scholl, Phys. Lett. B209 (1988) 434; B227 (1989) 227; 